\title{
Characterization of the Volatile Compounds of Zhenba Bacon at Different Process Stages Using GC-MS and GC-IMS
}

\author{
Linjie $\mathrm{Xi}^{1}{ }^{1}$, Jing Zhang ${ }^{2}$, Ruixiao $\mathrm{Wu}^{1}{ }^{1}$, Tian Wang ${ }^{1}$ and Wu Ding ${ }^{1, *}$ \\ 1 College of Food Science and Engineering, Northwest A\&F University, Xianyang 712100, China; \\ 15829031947@163.com (L.X.); Ruixiao_Wu@163.com (R.W.); sxsxys1997@163.com (T.W.) \\ 2 College of Horticulture, Northwest A\&F University, Xianyang 712100, China; yyzhj@nwsuaf.edu.cn \\ * Correspondence: ding_wu@nwsuaf.edu.cn; Tel.: +86-029-8709-2486
}

check for updates

Citation: Xi, L.; Zhang, J.; Wu, R.; Wang, T.; Ding, W. Characterization of the Volatile Compounds of Zhenba Bacon at Different Process Stages Using GC-MS and GC-IMS. Foods 2021, 10, 2869. https://doi.org/ $10.3390 /$ foods 10112869

Academic Editor: Manuel Juárez

Received: 26 October 2021

Accepted: 16 November 2021

Published: 19 November 2021

Publisher's Note: MDPI stays neutral with regard to jurisdictional claims in published maps and institutional affiliations.

Copyright: (c) 2021 by the authors. Licensee MDPI, Basel, Switzerland. This article is an open access article distributed under the terms and conditions of the Creative Commons Attribution (CC BY) license (https:// creativecommons.org/licenses/by/ $4.0 /)$.

\begin{abstract}
Zhenba bacon (ZB), a type of Chinese traditional bacon with a long history, has attracted considerable attention in the Southwest of China for its unique flavor. To elucidate the changing course of aroma components during the process of $\mathrm{ZB}$, four stages of process stages were assessed by GC-MS and GC-IMS coupled with multivariate data analysis. A total of 44 volatile compounds were identified by GC-IMS, including 5 esters, 8 alcohols, 12 aldehydes, 3 ketones, 1 furan and 2 sulfides; 40 volatile compounds were identified by GC-MS, 4 ketones, 7 phenols, 8 alcohols, 6 esters, 6 aldehydes, and 6 other compounds were detected. During the curing period, the amount and content of esters in Zhenba bacon gradually increased. Phenols appear in large quantities during the smoking period. The VOCs (volatile organic compounds) in the gallery plots were the most diverse in YZ samples, which are mainly esters. POV (peroxide value) and TBARS (thiobarbituric acid reactive substance) showed that lipid oxidation played an important role in the formation of volatile flavor components of bacon. This study provides valuable analytical data to explain the flavor formation of Zhenba bacon.
\end{abstract}

Keywords: Chinese bacon; volatile organic compounds; GC-MS; GC-IMS

\section{Introduction}

Chinese bacon belongs to a wide range of salted, dried, and smoked meat products available in China. Improvements in people's living standards lead to changes in their diets, and over the past few decades, smoked meat products have risen in popularity due to their unique flavor.

Zhenba bacon is typically processed for two months, beginning in November and ending in December, based on the lunar calendar, with traditional processing methods of salting and smoking. Zhenba is located in the Qinba mountains, with temperatures at $0 \sim 10{ }^{\circ} \mathrm{C}$ in the winter, making it a suitable climate for the production of bacon. At the same time, Zhenba bacon is a geographical symbol of China with an attractive color and a unique flavor, deeply favored by consumers.

The flavor of Chinese bacon is a complex process involving a series of chemical reactions and a variety of factors. At present, most scholars at home and abroad believe that the flavor mainly follows three pathways: lipid oxidation, degradation of the precursor, and the Maillard reaction. Lipid oxidation in the formation of flavor in meat products is mainly caused by lipid oxidation [1].

The research on lipid oxidation has examined two main areas [2,3]. The first area is, the oxidation of double bonds of unsaturated fatty acid to generate peroxides, which can be further broken down to volatile carbonyl compounds. Further, hydroxyl fatty acid is hydrolyzed to produce acids and the acids undergo dehydration and cyclization to yield the final lactone compounds with meat aromas.

A second aspect is that the degradation of precursor substances mainly includes the degradation of protein and oil [4]. Of course, the Maillard reaction is one of the most 
important reactions resulting from the heating process [5]. It utilizes the reaction between amino acids and reduces sugars to produce a variety of volatile flavor compounds in meat, such as pyrazine and pyridine [6].

At present, research on volatile flavor substances in smoked meat mainly uses gas chromatography-mass spectrometry (GC-MS), full two-dimensional gas chromatographytime-of-flight mass spectrometry (GC $\times$ GC-TOF MS), gas chromatography-sniffing (GC-O), electronic nose technology, etc. [7-11]. The gas chromatography-mass spectrometry (GC-IMS) ion migration technique has been emerging in recent years as a rapid detection of volatile flavor of advanced technology, GC-IMS can obtain the flavor substances and the composition of the discriminant information and sample quality, with high sensitivity, fast detection speed, easy operation and a sample analysis at low temperature, to better reflect the existing flavor state [12] of the sample. The GC-IMS approach was implemented to detect the flavor characteristics of food volatile compounds, which are usually employed to distinguish the odor characteristics of different samples. At present, the technique is commonly applied in meat [13,14], fruits [15,16], and wine [17]. Most of the previous studies mainly focused on the aroma and quality control and grading from food. GC-IMS has been widely used in food flavor analysis [18]. However, it is seldom used in Chinese bacon, which provides a new efficient method for future research on Chinese bacon, and can be combined with other detection techniques to obtain more suitable results.

In order to provide some theoretical reference for the deep processing of Zhenba bacon and the development of food dishes, the evolution of volatile flavor substances of Zhenba bacon in different processing periods was studied by utilizing GC-IMS technology combined with GC-MS and heatmap analysis.

\section{Materials and Methods}

\subsection{Sample Preparation}

For this study, fresh pork was purchased from a market in Zhenba, Hanzhong City, Shaanxi Province, China, and then samples were sent to the Mengerjie organic pork bacon factory (Zhenba) for processing.

Bacon was processed according to the traditional procedures of Zhenba bacon production. First, a volume of $1000 \mathrm{~mL}$ of baijiu (Chinese liquor) was uniformly spread on the surface of the pork, and then it was cured for 3 days with $4 \%$ dry salt $(\mathrm{g} / 100 \mathrm{~g}$ fresh sample) at $15-20^{\circ} \mathrm{C}$. After marination, all the samples were washed to remove the excess salt on the surface and then drained for a day. Finally, the samples were intermittently cold-smoked using Cyclobalanopsis glauca wood for 15 days until the meat was dried. The relative humidity during the processing depended on the climatic conditions. The different stages of Zhenba bacon (1000 g, $n=5$ each) were vacuum packed and kept frozen at $-80{ }^{\circ} \mathrm{C}$ until analyzed. All of the chemical reagents used in this study were of HPLC grade.

\subsection{Lipid Oxidation Analysis}

Lipid oxidation was assessed by measuring TBARS value and POV. TBARS were measured according to Jin et al. [19] with slight modifications. The POV of the lipid sample was determined following the method of Simsec and Kilic [20].

\subsection{GC-MS Analysis}

A head space solid-phase microextraction (HS-SPME) fiber assembly combined with a GC-MS system (GC-MS 2010 SE, SHIMADZU, Tokyo, Japan) was used for detecting volatile compounds from the selected samples. Briefly, bacon samples $(3 \mathrm{~g})$ were added into a headspace bottle for balance at $45^{\circ} \mathrm{C}$ for $30 \mathrm{~min}$, and an SPME fiber $(50 / 30 \mu \mathrm{m}$ CAR/PDMS/DVB extraction head, Supelco, PA, USA) was used to adsorb the volatile compounds from the vial headspace at $60^{\circ} \mathrm{C}$ for $30 \mathrm{~min}$. After sampling the fiber was transferred to a gas chromatography inlet and desorbed at $250^{\circ} \mathrm{C}$ for $3 \mathrm{~min}$. Compounds were separated in a DB-WAX capillary column $(30 \mathrm{~m} \times 0.25 \mathrm{~mm} \times 0.25 \mu \mathrm{m}$, Agilent $\mathrm{J} \& \mathrm{~W}$, Santa Clara, CA, USA). Helium (99.999\%) was used as carrier gas at a flow rate of $1 \mathrm{~mL} / \mathrm{min}$ 
and the injection volume was $1 \mu \mathrm{L}$. The temperature in the GC oven was maintained at $40{ }^{\circ} \mathrm{C}$ for $3 \mathrm{~min}$, and then raised to $90^{\circ} \mathrm{C}$ at a rate of $5^{\circ} \mathrm{C} \mathrm{min}{ }^{-1}$ and to $230{ }^{\circ} \mathrm{C}$ at a rate of $10^{\circ} \mathrm{C} \mathrm{min}{ }^{-1}$. The ion source was EI and the temperature was set at $200{ }^{\circ} \mathrm{C}$. The interface temperature was set at $250{ }^{\circ} \mathrm{C}$. A positive ionization potential of $\mathrm{MS}$ was at $70 \mathrm{eV}$, detection voltage of $1000 \mathrm{~V}$ and emission current of $100 \mu \mathrm{A}$. Semi-quantitative determination was obtained by using 2-methyl-3-heptanone as an internal standard. The volatile compounds acquired were identified for the reverse match factor (similarity $>700$ ) and retention index (RI). The C7 C30 n-alkanes were employed to calculate linear RI of volatile compounds. Five replicates were applied for each product.

\subsection{GC-IMS Analysis}

Bacon samples ( $2 \mathrm{~g}$ ) were placed into a $20 \mathrm{~mL}$ headspace (HS) vial and closed with a magnetic cap before analyzing. Subsequently, samples were incubated at $80{ }^{\circ} \mathrm{C}$ for $20 \mathrm{~min}$. After incubation, a constant head-space $(200 \mu \mathrm{L})$ was injected into the injector automatically by a heated syringe $\left(85^{\circ} \mathrm{C}\right)$. Then the samples were transferred into an MXT-5 (15 $\mathrm{m} \times$ $0.53 \mathrm{~mm}$ ) capillary column by nitrogen $(99.99 \%)$ at a programmed flow as follows: initially $2.0 \mathrm{~mL} / \mathrm{min}, 10 \mathrm{~mL}$ for $10 \mathrm{~min}, 100 \mathrm{~mL}$ for $10 \mathrm{~min}$ and eventually $150 \mathrm{~mL}$ for $10 \mathrm{~min}$. The ions of analytes ionized were directed to the drift tube with a constant temperature of $45^{\circ} \mathrm{C}$ and the drift gas (nitrogen gas, $99.99 \%$ purity) was set at $150 \mathrm{~mL} / \mathrm{min}$. The final results were the averages of three replicates.

\subsection{Statistical Analysis}

IMS data were analyzed by the instrumental analysis software including LAV (from G.A.S., Dortmund, Germany version 2.0.0), Reporter, Gallery Plot as well as GC × IMS Library Search, which can be used for sample analysis from different angles. The identified compounds were characterized combing retention index (RI) and drift time with NIST Library and IMS database retrieval software from G.A.S. The analysis software of the instrument includes VOCAL and three plugins, which can analyze samples from different angles.

\section{Results and Discussion}

\subsection{Lipid Oxidation}

Volatile compounds are mainly composed of lipid oxidation products. POV is the measure of hydroperoxides formed during autoxidation. POV represents the degree of primary oxidation of lipids. Hydroperoxides are not stable and decompose spontaneously to form other compounds, such as aldehydes, ketones, alcohols, acids [21]. The extent of lipid peroxidation is measured by the formation of TBARS. TBARS assay detects lipid peroxidation products. The TBARS' value refers to the reaction result of TBA with derivatives, such as malondialdehyde produced by oxidative decomposition of unsaturated fatty acids in animal fats. A high or low value indicates that the amount of secondary oxidation products, or final products, of fat has a strong correlation with the data of sensory analysis is one of the most widely used indexes to evaluate the degree of fat oxidation. Changes in POV and TBARS values at different processing stages of Zhenba bacon are shown in Figure 1. The treatments of control represent the four stages of: raw meat stage (fresh), curing stage (YZ), smoking stage (XZ), and mature stage (CP). Compared to the control (Fresh), the TBARS values of other groups significantly increased with processing time $(p<0.05)$, the TBARS value changes in bacon are due to the changes in lipid during the heating and smoking process. In general, TBARS' values increased with increasing process period, due to the promotion of lipid oxidation and dehydration. It indicates that the degree of secondary oxidation of lipids is gradually deepening. It can be seen from the figure that after the sample is pickled, the activity of lipoxidase is enhanced due to the increase of salt, thus the secondary oxidation of lipids is intensified. For the smoking stage, as TBARS values decreased slightly, the further oxidation of aldehydes to organic alcohols or carboxylic acids is inferred. 


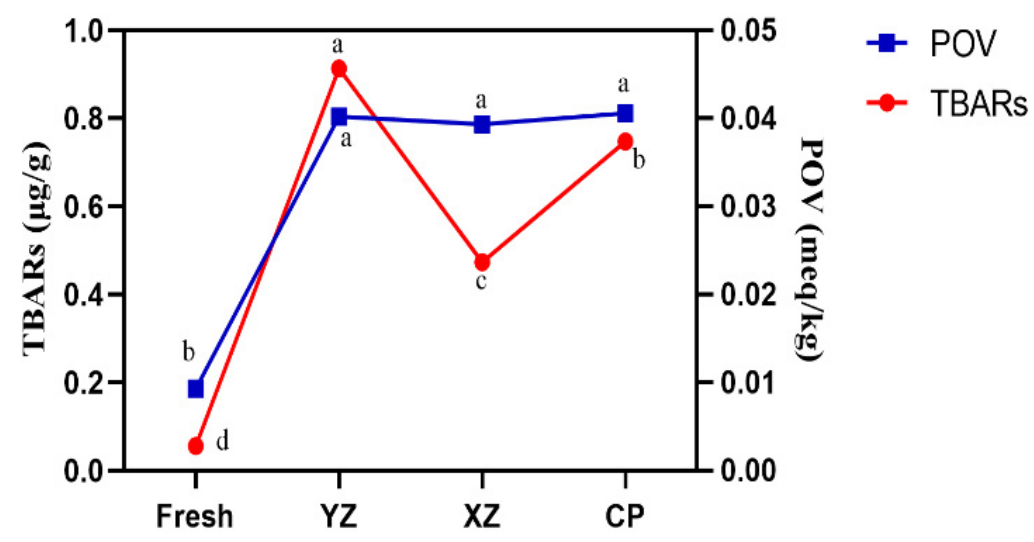

Figure 1. Changes of POV and TBARS content in different stages. Fresh: raw sample; YZ: cured sample; XZ: smoking sample; CP: finished sample; POV: peroxide value; TBARS: thiobarbituric acid reactive substance.

The trends in the POV are consistent with those of the TBARS change. During bacon processing, these values were increased. The POV value reflects the content of primary lipid oxidation products, indicating that the accumulation of primary oxidation products is basically the same during processing. Jin et al. [22] found that the TBARS are mainly aldehydes, which are then degraded into volatile compounds. POV and TBARS showed that under fermentation and smoking conditions, hydroperoxide and free fatty acids were produced from the lipids of Zhenba bacon, and the lipid hydrolysates played an important role in the formation of volatile flavor components of bacon.

\subsection{GC-IMS Topographic Plots}

The topographic plot map of the four stages of Zhenba bacon in GC-IMS is shown in Figure 2. The horizontal axis represents the migration time and the vertical axis represents each point on both sides of the reactive ion on peak (RIP peak) during retention of gas phase color spectrum representing a volatile organic matter. Colors represent the concentration of compounds; the white represents the low concentration and the red represents the high concentration. Meanwhile, deeper color indicates increased concentration. Most of the signals appeared in the retention time range from 100-400 $\mathrm{s}$ and drift time from 1.0-1.5 s. It can be seen from this plot that VOCs of samples at different stages are well separated from each other. The amounts of VOCs detected in the yellow box area of the fresh sample had the least content from Figure 2. The type and content of VOCs always increase with processing time. The VOCs in the red box area were the most diverse in YZ samples, while the differences were significant in other stage samples. From the GC-MS data, it is also evident that accumulation of the major constituents in $\mathrm{YZ}$ sample are esters. Figure 2 shows that the differences in VOCs of Zhenba bacon in different processing periods are mainly manifested in the position, number, intensity, and time of ion peaks. Compared with $\mathrm{CP}$ sample, the $\mathrm{XZ}$ sample is relatively close on the topographic map. Smoking led to the unique flavor, such as guaiacol. Combined with the data of GC-MS, it can be seen that a large number of phenolic substances are mainly produced. Meanwhile, the two stages only carried out a short burning and washing process, the influence on the change of flavor substances is less. In summary, compared with these samples, the signal intensities of substances are different among the four stages. As evident from the figure, VOCs of the different processing stages display significant differences in their topographic plots. Zhu et al. [23] found that fermentation can increase the VOCs, which can be seen intuitively in the topographic plot. Similarly, the results suggested that the identified number and concentration of VOCs in the processed samples were higher than that in the fresh samples. 


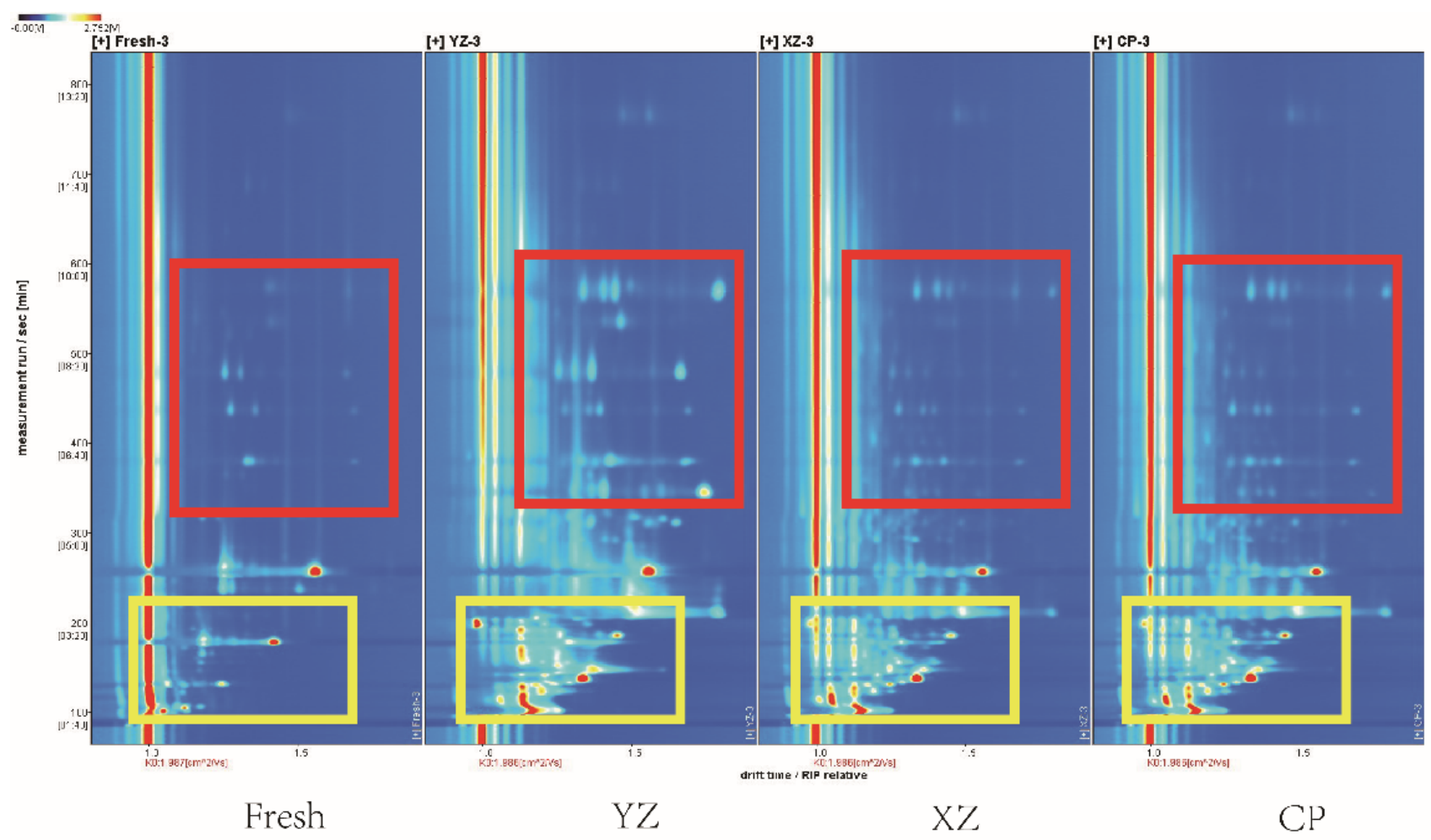

Figure 2. The gallery plots of VOCs from different stages using CC-IMS. Fresh: raw sample; YZ: cured sample; XZ: smoking sample; CP: finished sample; VOCs: volatile organic compounds.

\subsection{Analysis of VOCs by Fingerprint}

According to Figure 3, one can see that the concentration and kinds of VOCs of Zhenba bacon from four different processing stages are significantly different. It can be seen that the fresh stage contains substances that are either absent or at low concentrations in subsequent processing. The substances in region A mainly include acetone, pentanal, 1-pentanol, etc. In the flavor study, pentanal, the major straight-chain aliphatic aldehyde, contributes to the fruity and ester aromas. Due to its low flavor threshold, it could affect flavor or aroma in meat foods [24]. The variety and concentration of volatile substances were the highest in the $\mathrm{YZ}$ stage. The cured processing is involved in enzymatic hydrolysis of protein [25]. Therefore, it could contribute to the formation of bacon flavor. From left to right, substances in region B in the figure are mainly E-2-heptanal, 2, 3-butanedione, 2-methylpropanol, octanal, 3-methylbutanol, 1-propanol, ethyl hexanoate, heptanal, dimethyl disulphide, 3-methylthio-propanol etc. On the basis of structural considerations, it is assumed that 3-methylbutanal and 3-methylbutanol are derived from leucine while 2-methylbutanol is derived from isoleucine [26]. It is possible that these substances came from the local baijiu during the curing of bacon. 3-methylbutanol has a banana-like and pear-like aroma [27]. 2, 3-butanedione is found in numerous common flavors such as "buttery/creamy" and fruit flavors [28], and the smell is strong. It is speculated that this is the main flavor in the bacon curing period. The concentration of substances in the $\mathrm{C}$ region of $\mathrm{CP}$ sample rose to the highest, namely 2-butanone, ethyl propanoate, butanal, 2-methylbutanal and 3-methylbuanal, etc. Meanwhile, it was observed that among the four stages, $X Z$ and $C P$ were the most similar, while fresh and YZ had the biggest difference. Ethyl propanoate was associated with a green-sweet-fruity note [29], and butanal was the most abundant VOC in the meat samples [30]. 


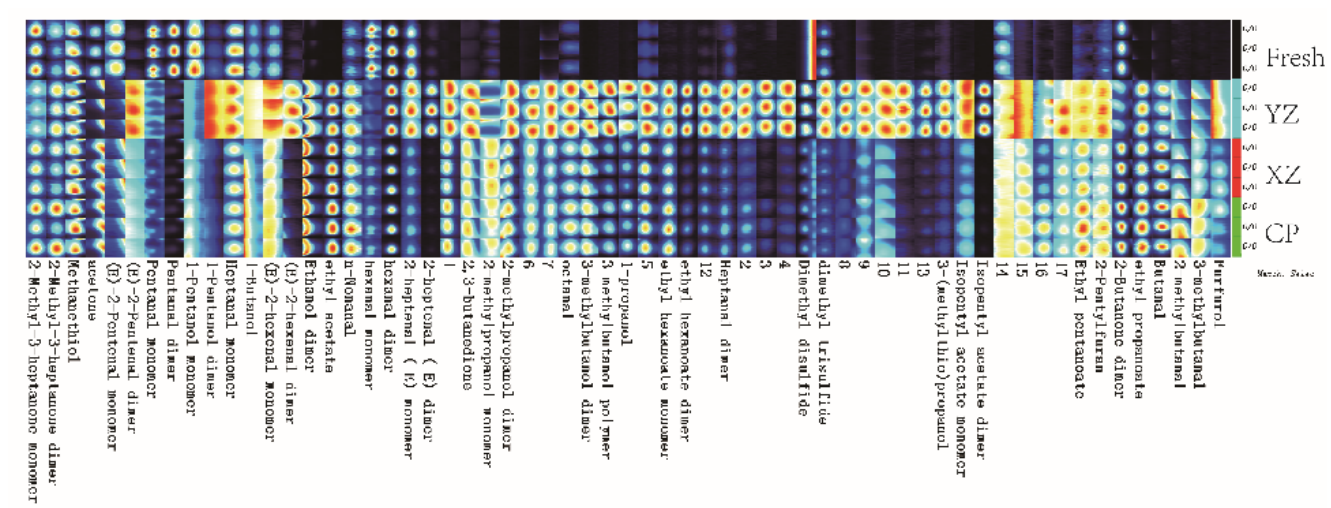

Figure 3. Fingerprints of VOCs isolated from different stages using GC-IMS. Each colored point represents a VOC, and different colors indicate varying concentrations, red representing higher intensity, blank meant not present. Fresh: raw sample; YZ: cured sample; XZ: smoking sample; CP: finished sample; VOCs: volatile organic compounds.

\subsection{Analysis of VOCs Obtained by GC-IMS and GC-MS}

According to the data from the company's built-in NIST gas phase retention index database and IMS migration time database, the volatile components were qualitatively analyzed according to the gas chromatography retention time and IMS migration time of volatile substances. A total of 44 volatile compounds were identified by GC-IMS, as detailed in Table 1, and included 5 esters, 8 alcohols, 12 aldehydes, 3 ketones, 1 furan and 2 sulfides. Thus, the volatile organic compounds in bacon are mainly aldehydes, alcohols, and esters. Due to the different concentrations of these compounds, some single compounds may produce multiple signals or spots (dimers or trimers) [31]. The retention indexes of the majority of the volatile compounds ranged from 200 to 400, which indicated that weakly polar and small-molecule volatile compounds were detected in the samples. This may be because the evaluation of advanced nondestructive testing of GC-IMS were similar to the sensory conditions of the samples [32]. Among the abovementioned compounds, 17 compounds, including acetone, 1-propanol, and 3-methylbutanal, were not detected by GC-MS. This suggested that GC-IMS technology can be used to comprehensively determine the real aroma of a sample without sample pretreatment [33]. In previous studies in Huangjiu [34], on sorghum [35], chicken [36], wheat [37], and kiwifruit [38], the differences of aroma components among samples were also revealed by GC-MS and GC-IMS.

In GC-MS, 40 volatile organic compounds, 4 ketones, 7 phenols, 8 alcohols, 6 esters, 6 aldehydes, and 6 other compounds were detected (Table 2). It could be clearly seen from Figure 4 that each color represents a different type of volatile compound, and the length represents the number of species. Among them, the fresh sample only exhibits one kind of VOCs (hexanal) and XZ sample had similar flavor substance types with the $\mathrm{CP}$ sample. The YZ sample has more alcohol compounds and aldehyde compounds. As one of the characteristic flavor products of fat degradation, aldehydes have a low threshold value and complex taste, so their contribution to the overall flavor of Zhenba bacon is more prominent, in particular, the impact of low carbohydrate aldehydes on the flavor of bacon is more significant. Among them, saturated straight chain aldehydes generally have a strong taste of raw oil and make people vomit, which is a major characteristic flavor substance in fresh meat. In addition, hexanal can be detected in every sample. As a common flavor substance in fresh meat, hexanal has been reported many times, and it has a grass-like taste [39]. In the fresh group, only one volatile flavor substance of hexanal was detected, and in the YZ group, the maximum value was 3.496. During the curing period, a total of five aldehydes were detected, namely pentanal, hexanal, 1-nonanal, 5-methyl furfural and acetal. Some studies have found that the appropriate amount of aldehyde substances (acetal) in liquor can improve the flavor of liquor, so acetal may be used in the liquor flavor during the $\mathrm{YZ}$ stage. The types and contents of aldehydes are the most similar in the smoked period and finished product period, which may be caused by the covalent 
combination of aldehydes and proteins. With the extension of storage time, aldehydes' flavor substances are not volatile.

Table 1. The information on detected VOCs of Zhenba bacon by GC-IMS.

\begin{tabular}{|c|c|c|c|c|c|c|c|c|}
\hline Count & Compound & CAS ${ }^{\#}$ & Formula & $\mathrm{MW}^{\mathrm{a}}$ & $\mathbf{R I}^{b}$ & $\mathrm{Rt}^{\mathrm{c}}[\mathrm{Sec}]$ & $D t^{d}$ & Comment \\
\hline 1 & Methanethiol & C74931 & $\mathrm{CH} 4 \mathrm{~S}$ & 48.1 & 504.1 & 96.119 & 1.03955 & \\
\hline 2 & Ethyl Acetate & C141786 & $\mathrm{C} 4 \mathrm{H} 8 \mathrm{O} 2$ & 88.1 & 597.9 & 137.091 & 1.34375 & \\
\hline 3 & 3-Methylbutanal & C590863 & C5H10O & 86.1 & 645.1 & 157.712 & 1.41113 & \\
\hline 4 & 2-Methylbutanal & C96173 & $\mathrm{C} 5 \mathrm{H} 10 \mathrm{O}$ & 86.1 & 656.3 & 162.596 & 1.40419 & \\
\hline 5 & Pentanal & C110623 & $\mathrm{C} 5 \mathrm{H} 10 \mathrm{O}$ & 86.1 & 692.3 & 179.691 & 1.1862 & monomer \\
\hline 6 & Pentanal & C110623 & $\mathrm{C} 5 \mathrm{H} 10 \mathrm{O}$ & 86.1 & 693.7 & 180.776 & 1.42797 & dimer \\
\hline 7 & Ethyl Hexanoate & $\mathrm{C} 123660$ & $\mathrm{C} 8 \mathrm{H} 16 \mathrm{O} 2$ & 144.2 & 1002.1 & 568.154 & 1.33495 & monomer \\
\hline 8 & Ethyl Hexanoate & $\mathrm{C} 123660$ & $\mathrm{C} 8 \mathrm{H} 16 \mathrm{O} 2$ & 144.2 & 1002.5 & 568.932 & 1.80397 & dimer \\
\hline 9 & Heptanal & C111717 & $\mathrm{C} 7 \mathrm{H} 14 \mathrm{O}$ & 114.2 & 898.4 & 382.978 & 1.32916 & monomer \\
\hline 10 & Heptanal & C111717 & $\mathrm{C} 7 \mathrm{H} 14 \mathrm{O}$ & 114.2 & 896.2 & 379.088 & 1.69106 & dimer \\
\hline 11 & 2-Heptenal (E) & C18829555 & $\mathrm{C} 7 \mathrm{H} 12 \mathrm{O}$ & 112.2 & 951.9 & 477.9 & 1.25967 & monomer \\
\hline 12 & 2-Heptenal (E) & C18829555 & $\mathrm{C} 7 \mathrm{H} 12 \mathrm{O}$ & 112.2 & 952.7 & 479.456 & 1.67369 & dimer \\
\hline 13 & Acetone & C67641 & $\mathrm{C} 3 \mathrm{H} 6 \mathrm{O}$ & 58.1 & 530.9 & 107.842 & 1.12549 & \\
\hline 14 & 1-Propanol & C71238 & $\mathrm{C} 3 \mathrm{H} 8 \mathrm{O}$ & 60.1 & 569.6 & 124.745 & 1.25108 & \\
\hline 15 & 2,3-Butanedione & C431038 & $\mathrm{C} 4 \mathrm{H} 6 \mathrm{O} 2$ & 86.1 & 586.2 & 132.008 & 1.17563 & \\
\hline 16 & Dimethyl Disulfide & C624920 & C2H6S2 & 94.2 & 716.9 & 199.324 & 0.98008 & \\
\hline 17 & 1-Pentanol & C71410 & $\mathrm{C} 5 \mathrm{H} 12 \mathrm{O}$ & 88.1 & 766.6 & 238.992 & 1.24972 & monomer \\
\hline 18 & 1-Pentanol & C71410 & $\mathrm{C} 5 \mathrm{H} 12 \mathrm{O}$ & 88.1 & 766.1 & 238.594 & 1.51116 & dimer \\
\hline 19 & Furfurol & C98011 & $\mathrm{C} 5 \mathrm{H} 4 \mathrm{O} 2$ & 96.1 & 820.9 & 293.414 & 1.34139 & \\
\hline 20 & (E)-2-Hexenal & C6728263 & $\mathrm{C} 6 \mathrm{H} 10 \mathrm{O}$ & 98.1 & 844 & 318.919 & 1.18372 & monomer \\
\hline 21 & (E)-2-Hexenal & C6728263 & $\mathrm{C} 6 \mathrm{H} 10 \mathrm{O}$ & 98.1 & 842.7 & 317.4 & 1.5273 & dimer \\
\hline 22 & 2-Pentylfuran & C3777693 & $\mathrm{C} 9 \mathrm{H} 14 \mathrm{O}$ & 138.2 & 990.1 & 545.855 & 1.24933 & \\
\hline 23 & Ethanol & C64175 & $\mathrm{C} 2 \mathrm{H} 6 \mathrm{O}$ & 46.1 & 520.2 & 103.167 & 1.04743 & monomer \\
\hline 24 & Ethanol & C64175 & $\mathrm{C} 2 \mathrm{H} 6 \mathrm{O}$ & 46.1 & 522.2 & 104.023 & 1.17009 & dimer \\
\hline 25 & 2-Butanone & C78933 & $\mathrm{C} 4 \mathrm{H} 8 \mathrm{O}$ & 72.1 & 586.5 & 132.112 & 1.06086 & monomer \\
\hline 26 & 2-Butanone & C78933 & $\mathrm{C} 4 \mathrm{H} 8 \mathrm{O}$ & 72.1 & 587.8 & 132.685 & 1.24907 & dimer \\
\hline 27 & Hexanal & C66251 & $\mathrm{C} 6 \mathrm{H} 12 \mathrm{O}$ & 100.2 & 789.6 & 258.95 & 1.25527 & monomer \\
\hline 28 & Hexanal & C66251 & $\mathrm{C} 6 \mathrm{H} 12 \mathrm{O}$ & 100.2 & 788.8 & 258.092 & 1.56034 & dimer \\
\hline 29 & 2-Methylpropanol & C78831 & $\mathrm{C} 4 \mathrm{H} 10 \mathrm{O}$ & 74.1 & 624.8 & 148.857 & 1.16607 & monomer \\
\hline 30 & 2-Methylpropanol & C78831 & $\mathrm{C} 4 \mathrm{H} 10 \mathrm{O}$ & 74.1 & 624.8 & 148.857 & 1.36238 & dimer \\
\hline 31 & (E)-2-Pentenal & $\mathrm{C} 1576870$ & $\mathrm{C} 5 \mathrm{H} 8 \mathrm{O}$ & 84.1 & 744.1 & 220.997 & 1.10807 & monomer \\
\hline 32 & (E)-2-Pentenal & C1576870 & $\mathrm{C} 5 \mathrm{H} 8 \mathrm{O}$ & 84.1 & 746.2 & 222.68 & 1.35897 & dimer \\
\hline 33 & 1-Butanol & C71363 & $\mathrm{C} 4 \mathrm{H} 10 \mathrm{O}$ & 74.1 & 664.2 & 166.036 & 1.179 & \\
\hline 34 & 3-Methylbutanol & C123513 & $\mathrm{C} 5 \mathrm{H} 12 \mathrm{O}$ & 88.1 & 736.6 & 215.045 & 1.49618 & dimer \\
\hline 35 & 3-Methylbutanol & C123513 & $\mathrm{C} 5 \mathrm{H} 12 \mathrm{O}$ & 88.1 & 731.4 & 210.897 & 1.79254 & polymer \\
\hline 36 & Isopentyl Acetate & C123922 & $\mathrm{C} 7 \mathrm{H} 14 \mathrm{O} 2$ & 130.2 & 869.9 & 347.375 & 1.30617 & monomer \\
\hline 37 & Isopentyl Acetate & C123922 & $\mathrm{C} 7 \mathrm{H} 14 \mathrm{O} 2$ & 130.2 & 869.5 & 346.96 & 1.75 & dimer \\
\hline 38 & Ethyl Propanoate & C105373 & $\mathrm{C} 5 \mathrm{H} 10 \mathrm{O} 2$ & 102.1 & 701.9 & 187.341 & 1.45434 & \\
\hline 39 & Butanal & $\mathrm{C} 123728$ & $\mathrm{C} 4 \mathrm{H} 8 \mathrm{O}$ & 72.1 & 592 & 134.505 & 1.29064 & \\
\hline 40 & Dimethyl Trisulfide & C3658808 & $\mathrm{C} 2 \mathrm{H} 6 \mathrm{~S} 3$ & 126.3 & 954.7 & 482.904 & 1.31729 & \\
\hline 41 & 3-(Methylthio) Propanol & C505102 & C4H10OS & 106.2 & 984.9 & 536.605 & 1.464 & \\
\hline 42 & Octanal & C124130 & $\mathrm{C} 8 \mathrm{H} 16 \mathrm{O}$ & 128.2 & 1005.1 & 574.034 & 1.40534 & \\
\hline 43 & n-Nonanal & C124196 & $\mathrm{C} 9 \mathrm{H} 18 \mathrm{O}$ & 142.2 & 1106.2 & 770.91 & 1.47695 & \\
\hline 44 & Ethyl Pentanoate & C539822 & $\mathrm{C} 7 \mathrm{H} 14 \mathrm{O} 2$ & 130.2 & 896.9 & 380.317 & 1.27255 & \\
\hline $45^{*}$ & 2-Methyl-3-Heptanone & C13019200 & $\mathrm{C} 8 \mathrm{H} 16 \mathrm{O}$ & 128.2 & 930.7 & 440.309 & 1.27858 & monomer \\
\hline $46^{*}$ & 2-Methyl-3-Heptanone & C13019200 & $\mathrm{C} 8 \mathrm{H} 16 \mathrm{O}$ & 128.2 & 930.7 & 440.309 & 1.69262 & dimer \\
\hline
\end{tabular}

${ }^{a}$ Represents the molecular mass; ${ }^{b}$ Represents the retention index calculated using n-ketones C4-C9 as external standard on MXT-5 column;

${ }^{\mathrm{c}}$ Represents the retention time in the capillary GC column; ${ }^{\mathrm{d}}$ Represents the drift time in the drift tube; ${ }^{*}$ Internal standard substance;

\# Represents the Chemical Abstracts Service. 
Table 2. Volatile compounds in four stages of Zhenba bacon by GC-MS.

\begin{tabular}{|c|c|c|c|c|c|c|c|}
\hline Compound & Number & CAS & RI & Fresh & $\mathrm{YZ}$ & $\mathbf{X Z}$ & $\mathrm{CP}$ \\
\hline \multicolumn{8}{|l|}{ Ketones } \\
\hline 3-Methyl-1,2-Cyclopentanedione & M1 & $765-70-8$ & 1631.1 & - & - & $0.449 \pm 0.11$ & $0.329 \pm 0.14$ \\
\hline 3-Methyl-2-Cyclopenten-1-One & M2 & $2758-18-1$ & 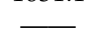 & - & - & $0.275 \pm 0.21$ & -1 \\
\hline 2,3-Octadione & M3 & $585-25-1$ & 1334.7 & - & $0.287 \pm 0.23$ & - & - \\
\hline 2,3-Dimethyl-2-Cyclopentene-1-One & M4 & $1121-05-7$ & 1538.1 & - & $0.078 \pm 0.02$ & $\longrightarrow$ & $\longrightarrow$ \\
\hline \multicolumn{8}{|l|}{ Phenols } \\
\hline 2,5-Dimethylphenol & M5 & $576-26-1$ & 2099.1 & - & - & - & $0.209 \pm 0.09$ \\
\hline 2-Methylphenol & M6 & $95-48-7$ & 2022.7 & - & - & $0.487 \pm 0.13$ & $0.362 \pm 0.17$ \\
\hline 3-Methylphenol & M7 & $108-39-4$ & - & - & - & $0.599 \pm 0.26$ & $0.696 \pm 0.22$ \\
\hline 4-Methyl Guaiacol & M8 & $93-51-6$ & 1694.0 & - & - & $0.522 \pm 0.03$ & $0.415 \pm 0.11$ \\
\hline 4-Ethylguaiacol & M9 & $2785-89-9$ & 2059.9 & - & - & $0.279 \pm 0.12$ & $0.221 \pm 0.03$ \\
\hline Guaiacol & M10 & $90-5-1$ & 1647.7 & - & - & $0.735 \pm 0.32$ & $0.567 \pm 0.27$ \\
\hline Phenol & M11 & $108-95-2$ & 2028.9 & - & - & $1.136 \pm 0.46$ & $0.869 \pm 0.29$ \\
\hline \multicolumn{8}{|l|}{ Alcohols } \\
\hline Ethanol & M12 & $64-17-5$ & 939.6 & - & $16.017 \pm 3.74$ & $3.982 \pm 1.01$ & $7.084 \pm 2.32$ \\
\hline Phenethyl Alcohol & M13 & $60-12-8$ & 1673.0 & - & $0.135 \pm 0.08$ & - & - \\
\hline 3-Octenol & M14 & $3391-86-4$ & 1627.4 & & $0.365 \pm 0.43$ & $0.129 \pm 0.03$ & $0.214 \pm 0.01$ \\
\hline Furfuryl Alcohol & M15 & $98-00-0$ & 1588.3 & - & - & $0.320 \pm 0.09$ & $0.241 \pm 0.01$ \\
\hline 2-Methyl-1-Propanol & M16 & $78-83-1$ & 1098.2 & . & $0.489 \pm 0.23$ & - & $0.103 \pm 0.01$ \\
\hline 3-Methyl-1-Butanol & M17 & $123-51-3$ & 1214.0 & - & $5.757 \pm 1.51$ & $0.83 \pm 0.03$ & $1.643 \pm 0.16$ \\
\hline 1-Hexanol & M18 & $111-27-3$ & 1360.1 & - & $0.146 \pm 0.05$ & - & - \\
\hline 1-Pentanol & M19 & $71-41-0$ & 1256.3 & - & $0.332 \pm 0.07$ & $0.083 \pm 0.01$ & $0.063 \pm 0.03$ \\
\hline \multicolumn{8}{|l|}{ Esters } \\
\hline Ethyl Acetate & M20 & $141-78-6$ & 888.0 & & - & $0.193 \pm 0.01$ & \\
\hline Ethyl Octanoate & M21 & $106-32-1$ & 1606.4 & 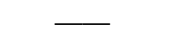 & - & - & $0.091 \pm 0.02$ \\
\hline Ethyl Valerate & M22 & $539-82-2$ & 1129.4 & & $0.187 \pm 0.03$ & - & - \\
\hline Ethyl Caproate & M23 & $123-66-0$ & 1241.8 & - & $1.131 \pm 0.29$ & $0.258 \pm 0.11$ & $0.311 \pm 0.01$ \\
\hline Methyl Hexanoate & M24 & $106-70-7$ & 1194.4 & - & $0.718 \pm 0.37$ & $0.790 \pm 0.35$ & $0.614 \pm 0.19$ \\
\hline Ethyl Caprate & M25 & $110-38-3$ & 1574.6 & & + & $0.191 \pm 0.01$ & - \\
\hline Ethyl Butyrate & M26 & $105-54-4$ & 1041.8 & & $0.342 \pm 0.02$ & $0.102 \pm 0.02$ & $0.168 \pm 0.03$ \\
\hline Methyl Butyrate & M27 & $623-42-7$ & 990.1 & & - & $0.460 \pm 0.27$ & $0.375 \pm 0.11$ \\
\hline Ethyl Lactate & M28 & $97-64-3$ & 1356.9 & $\bar{L}$ & $0.104 \pm 0.03$ & - & $0.082 \pm 0.03$ \\
\hline \multicolumn{8}{|l|}{ Aldehydes } \\
\hline Pentanal & M29 & $110-62-3$ & 986.6 & - & $0.112 \pm 0.01$ & - & 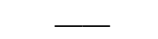 \\
\hline Hexanal & M30 & $66-25-1$ & 1091.0 & $0.203 \pm 0.03$ & $3.496 \pm 0.85$ & $0.834 \pm 0.17$ & $0.892 \pm 0.26$ \\
\hline 1-Nonanal & M31 & $124-19-6$ & 1419.2 & - & $0.323 \pm 0.03$ & $0.203 \pm 0.03$ & $0.153 \pm 0.01$ \\
\hline Furfural & M32 & $98-1-1$ & 1680.2 & - & - & $0.762 \pm 0.21$ & $0.562 \pm 0.01$ \\
\hline 5-Methyl Furfural & M33 & $620-02-0$ & 1552.3 & & $0.760 \pm 0.01$ & $0.244 \pm 0.03$ & $0.173 \pm 0.01$ \\
\hline Acetal & M34 & $105-57-7$ & 893.2 & & $0.458 \pm 0.03$ & - & - \\
\hline \multicolumn{8}{|l|}{ Others } \\
\hline 1,4-Dimethoxybenzene & M35 & $150-78-7$ & 1767.6 & - & $0.158 \pm 0.01$ & $0.114 \pm 0.02$ & $0.185 \pm 0.03$ \\
\hline Naphthalene & M36 & $91-20-3$ & 1784.9 & - & - & $0.105 \pm 0.01$ & - \\
\hline 2-Acetylfuran & M37 & $1192-62-7$ & 1516.7 & & $0.326 \pm 0.02$ & $0.114 \pm 0.02$ & - \\
\hline Acetic Acid Glacial & M38 & $64-19-7$ & 1640.4 & - & $0.742 \pm 0.13$ & $0.621 \pm 0.24$ & $0.448 \pm 0.09$ \\
\hline Pyridine & M39 & $110-86-1$ & 1200.5 & - & - & $0.389 \pm 0.01$ & - \\
\hline Butyric Acid & M40 & $107-92-6$ & 1570.6 & - & - & $0.104 \pm 0.01$ & - \\
\hline
\end{tabular}

RI: Retention indexes; Represents the Chemical Abstracts Service; Fresh: raw sample; YZ: cured sample; XZ: smoking sample; CP: finished sample.

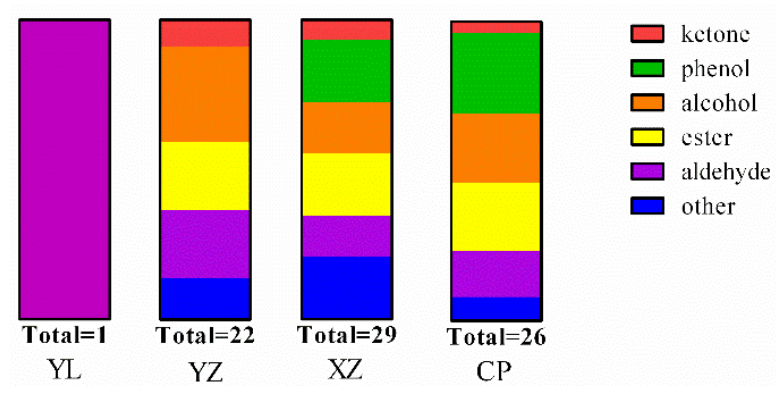

Figure 4. Change of VOCs species of Zhenba bacon with different stages using GC-MS. Fresh: raw sample; YZ: cured sample; XZ: smoking sample; CP: finished sample; VOCs: volatile organic compounds. 
Ketone was a product of lipid oxidation and hence was used as an indicator of lipid peroxidation [40]. 2,3-butanedione is a byproduct of the Maillard reaction. Because its odor threshold is low, it is likely to be an important contributor to provide buttery aroma with bacon. In the four stages, the content of 2,3-butanedione was observed only in the $\mathrm{YZ}$ stage. In this study, 3-Methyl-1,2-Cyclopentanedione was detected in XZ and CP stages. These methyl ketones, which are caused by autoxidation and $\beta$ oxidation of fatty acids or microbial metabolism, are considered to be important contributors to the complex flavor and aroma properties of pork [41].

Most alcohols are produced by the degradation of unsaturated fats, and some may be produced by the reduction of aldehydes. In the $\mathrm{XZ}$ and $\mathrm{CP}$ samples, the concentration of 3-octenol (grass aroma and mushroom aroma) [42] and furfuryl alcohol (mildew aroma and caramel aroma) indicated a significant contribution to the bacon flavor. 3-octenol has been associated with the pathway of arachidonic acid. The content of ethanol, 1-pentanol, and 3-methyl-1-butanol has a high level in $\mathrm{YZ}$, and a low level in $\mathrm{XZ}$ and $\mathrm{CP}$ samples. This could be due to the large contribution of liquor flavor substances in the $Y Z$ stage. Previous studies have shown that linear alcohols were known to originate from lipid oxidation; branched-chain alcohols were produced by a Millard-type reaction and Strecker degradation under fermentation conditions and then reduced [43].

The proportion of esters in the volatile components of Zhenba bacon was the largest, which contributed to the flavor of bacon. The formation of esters usually requires a complex reaction chain, which may come from the esterification of alcohols and carboxylic acids under the action of microorganisms [44]. Among the identified substances of esters were ethyl valerate, ethyl caproate, methyl hexanoate, ethyl butyrate, and ethyl lactate. However, content of ethyl caproate is the highest in the $\mathrm{YZ}$ stage, which is mainly produced in the fermentation process, and has the aroma of pineapple and banana fruits [45].

Phenolic substances are mainly generated by the decomposition of lignin in fumigating materials, which are the unique volatile flavor substances of smoked products. It is attributed mainly to the volatile compounds generated during the heating and decomposing of the smoked material, and most of the phenolic substances formed after being smoked. The findings from the present study are consistent with this idea. Phenols are only present during the $\mathrm{XZ}$ and $\mathrm{CP}$ samples, which contained 2-methylphenol, 3-methylphenol, 4-methyl guaiacol, 4-ethylguaiacol, guaiacol, and phenol. Phenolic did not differ much between these two stages.

\subsection{Correlation between GC-MS and Lipid}

Lipids were one of essential flavor precursors and taste contributors that affect meat flavor and palatability. Proper lipid oxidation is a major cause for changes of flavor during storage and processing [42]. The volatile compounds are mainly produced by lipid oxidation [21]. The TBARS' value is an index of secondary lipid oxidation, the POV value is an index of primary oxidation. Correlation analysis was used to explore the impacts of volatile flavor compounds of the Zhenba bacon during different stages and the lipid oxidation (Figure 5). A positive correlation was observed between lipid oxidation and some VOCs. Alcohols include ethanol, phenethyl alcohol, 3-octenol, 2-methyl-1-propanol, 3-methyl-1-butanol, 1-hexanol, and 1-pentanol. Aldehydes include pentanal, hexanal, 1-nonanal, 5-methyl furfural, and acetal. Esters include ethyl acetate, ethyl valerate, ethyl caproate, methyl hexanoate, and ethyl lactate. 


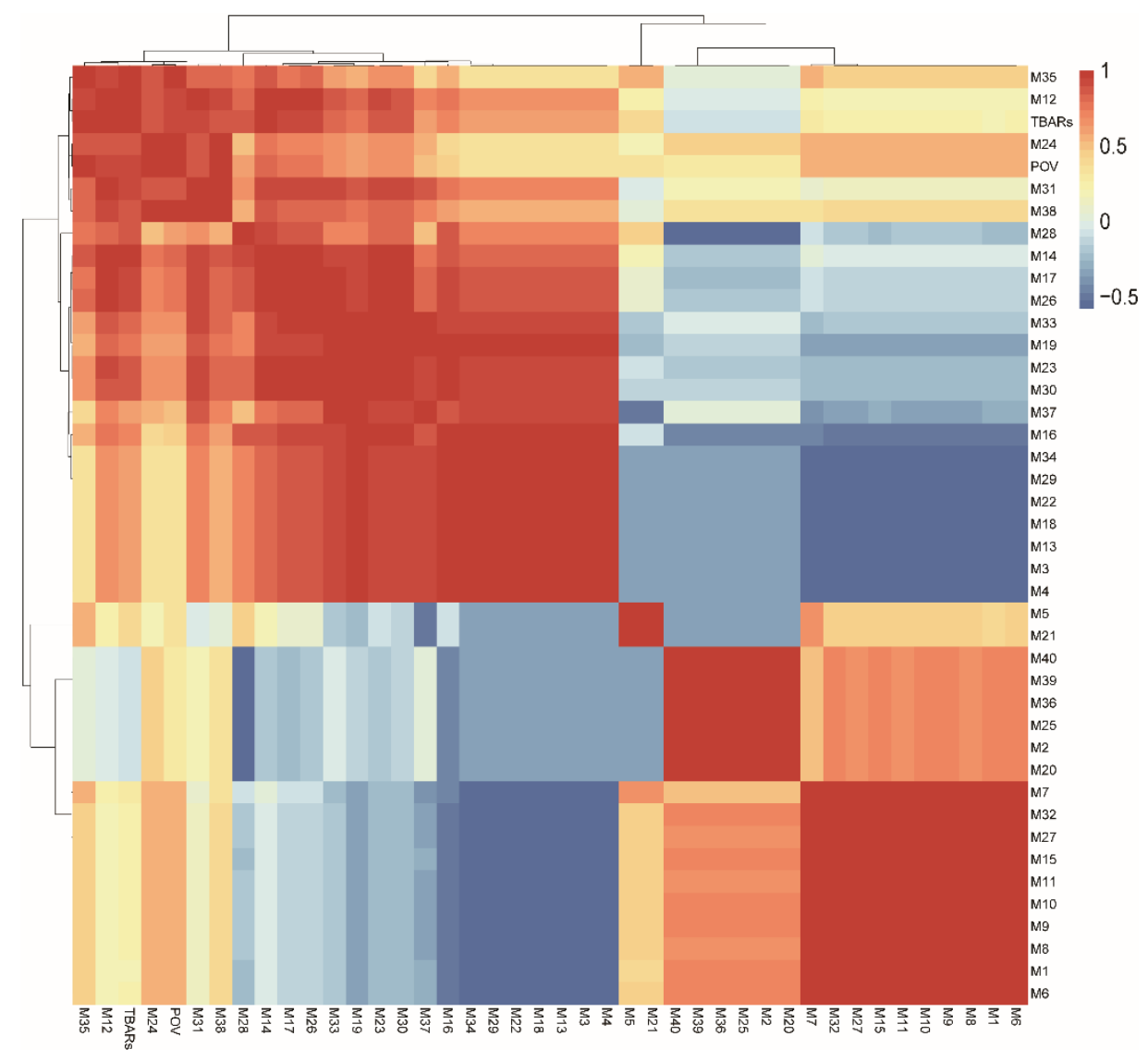

Figure 5. Correlations among POV, TBARS and GC-MS from different stages. The color from blue to red represents a correlation from negative to positive. Fresh: raw sample; YZ: cured sample; XZ: smoking sample; CP: finished sample; POV: peroxide value; TBARS: thiobarbituric acid reactive substance.

\section{Conclusions}

This study investigated the VOCs found in the four processing stages of Zhenba bacon. A total of 40 and 44 VOCs were identified in the four samples by GC-MS and GC-IMS. The volatile organic compounds in Zhenba bacon are mainly aldehydes, alcohols, and esters. The combined use of GC-MS and GC-IMS maximizes the results by combining accurate analytical results with intuitive visualization. There were two conspicuous differences between $\mathrm{YZ}$ and $\mathrm{XZ}$, which were the smoky attribute and lipid reaction. At the same time, esters increased in the $\mathrm{YZ}$ stage and phenolic compounds were derived from the $\mathrm{XZ}$ stage. POV and TBARS showed that under fermentation and smoking conditions, hydroperoxide and free fatty acids were produced from the lipids of Zhenba bacon, and the lipid hydrolysates played an important role in the formation of volatile flavor components of bacon. The results of this study support the view that smoking and curing are the most important stages. This research provides a necessary study of the flavor formation in Chinese bacon.

Author Contributions: Conceptualization, L.X. and W.D.; methodology, L.X.; software, L.X. and T.W.; validation, L.X., J.Z. and W.D.; formal analysis, R.W. and T.W.; investigation, W.D.; resources, W.D.; data curation, L.X.; writing—original draft preparation, L.X.; writing—review and editing, L.X.; visualization, L.X.; supervision, J.Z.; project administration, W.D.; funding acquisition, W.D. All authors have read and agreed to the published version of the manuscript.

Funding: This work was supported by the Study on Nano antisepsis and quality control technology of Meat (2021 NY-153).

Institutional Review Board Statement: Not applicable. 
Informed Consent Statement: Not applicable.

Conflicts of Interest: The authors declare no conflict of interest.

\section{References}

1. Berdague, J.L.; Monteil, P.; Montel, M.C.; Talon, R. Effects of starter cultures on the formation of flavor compounds in dry sausage. Meat Sci. 1993, 35, 275-287. [CrossRef]

2. Gandemer, G. Dry cured ham quality as related to lipid quality of raw material and lipid changes during processing: A review. Grasas Aceites 2009, 60, 297-307.

3. Harkouss, R.; Astruc, T.; Lebert, A.; Gatellier, P.; Loison, O.; Safa, H.; Portanguen, S.; Parafita, E.; Mirade, P.-S. Quantitative study of the relationships among proteolysis, lipid oxidation, structure and texture throughout the dry-cured ham process. Food Chem. 2015, 166, 522-530. [CrossRef] [PubMed]

4. Wannenmacher, J.; Gastl, M.; Becker, T. Phenolic Substances in Beer: Structural Diversity, Reactive Potential and Relevance for Brewing Process and Beer Quality. Compr. Rev. Food Sci. Food Saf. 2018, 17, 953-988. [CrossRef]

5. Li, M.; Yang, R.; Zhang, H.; Wang, S.; Chen, D.; Lin, S. Development of a flavor fingerprint by HS-GC-IMS with PCA for volatile compounds of Tricholoma matsutake Singer. Food Chem. 2019, 290, 32-39. [CrossRef] [PubMed]

6. Raza, A.; Song, H.; Begum, N.; Raza, J.; Iftikhar, M.; Li, P.; Li, K. Direct Classification of Volatile Organic Compounds in Heat-Treated Glutathione-Enriched Yeast Extract by Headspace-Gas Chromatography-Ion Mobility Spectrometry (HS-GC-IMS). Food Anal. Methods 2020, 13, 2279-2289. [CrossRef]

7. Hu, K.; Chang, R.; Zhu, Q.; Wan, J.; Tang, P.; Liu, C.; Song, L.; He, L.; Ye, C.; Zeng, X.; et al. Exploring the Mechanism of Liquid Smoke and Human Taste Perception Based on the Synergy of the Electronic Tongue, Molecular Docking, and Multiple Linear Regression. Food Biophys. 2020, 15, 482-494. [CrossRef]

8. Perez-Rodriguez, M.L.; Garcia-Mata, M.; Bosch-Bosch, N. Effect of adding smoke-flavouring to frankfurters on nitrite and nitrate levels. Food Chem. 1998, 62, 201-205. [CrossRef]

9. Saldana, E.; Saldarriaga, L.; Cabrera, J.; Siche, R.; Behrens, J.H.; Selani, M.M.; de Almeida, M.A.; Silva, L.D.; Silva Pinto, J.S.; Contreras-Castillo, C.J. Relationship between volatile compounds and consumer-based sensory characteristics of bacon smoked with different Brazilian woods. Food Res. Int. 2019, 119, 839-849. [CrossRef]

10. Samant, S.S.; Crandall, P.G.; O’Bryan, C.A.; Lingbeck, J.M.; Martin, E.M.; Tokar, T.; Seo, H.-S. Effects of smoking and marination on the sensory characteristics of cold-cut chicken breast filets: A pilot study. Food Sci. Biotechnol. 2016, 25, 1619-1625. [CrossRef]

11. Simko, P. Determination of polycyclic aromatic hydrocarbons in smoked meat products and smoke flavouring food additives. $J$. Chromatogr. B-Anal. Technol. Biomed. Life Sci. 2002, 770, 3-18. [CrossRef]

12. Martin-Gomez, A.; Arroyo-Manzanares, N.; Rodriguez-Estevez, V.; Arce, L. Use of a non-destructive sampling method for characterization of Iberian cured ham breed and feeding regime using GC-IMS. Meat Sci. 2019, 152, 146-154. [CrossRef]

13. Chen, M.; Chen, T.; Qi, X.; Lu, D.; Chen, B. Analyzing changes of volatile components in dried pork slice by gas chromatographyion mobility spectroscopy. CyTA-J. Food 2020, 18, 328-335. [CrossRef]

14. Chen, Y.P.; Cai, D.; Li, W.; Blank, I.; Liu, Y. Application of gas chromatography-ion mobility spectrometry (GC-IMS) and ultrafast gas chromatography electronic-nose (uf-GC E-nose) to distinguish four Chinese freshwater fishes at both raw and cooked status. J. Food Biochem. 2021, e13840. [CrossRef]

15. Yang, L.; Liu, J.; Wang, X.; Wang, R.; Ren, F.; Zhang, Q.; Shan, Y.; Ding, S. Characterization of Volatile Component Changes in Jujube Fruits during Cold Storage by Using Headspace-Gas Chromatography-Ion Mobility Spectrometry. Molecules 2019, $24,3904$. [CrossRef]

16. Yang, F.; Liu, Y.; Wang, B.; Song, H.; Zou, T. Screening of the volatile compounds in fresh and thermally treated watermelon juice via headspace-gas chromatography-ion mobility spectrometry and comprehensive two-dimensional gas chromatographyolfactory-mass spectrometry analysis. Lwt-Food Sci. Technol. 2021, 137. [CrossRef]

17. Zhou, R.; Chen, X.; Xia, Y.; Chen, M.; Zhang, Y.; Li, Q.; Zhen, D.; Fang, S. Research on the application of liquid-liquid extraction-gas chromatography-mass spectrometry (LLE-GC-MS) and headspace-gas chromatography-ion mobility spectrometry (HS-GC-IMS) in distinguishing the Baiyunbian aged liquors. Int. J. Food Eng. 2021, 17, 83-96. [CrossRef]

18. Xiao, O.; Li, M.; Chen, J.; Li, R.; Quan, R.; Zhang, Z.; Kong, Z.; Dai, X. Influence of Triazole Pesticides on Wine Flavor and Quality Based on Multidimensional Analysis Technology. Molecules 2020, 25, 5596. [CrossRef]

19. Jin, G.; Zhang, J.; Yu, X.; Zhang, Y.; Lei, Y.; Wang, J. Lipolysis and lipid oxidation in bacon during curing and drying-ripening. Food Chem. 2010, 123, 465-471. [CrossRef]

20. Simsek, A.; Kilic, B. Influences of encapsulated polyphosphate incorporation on oxidative stability and quality characteristics of ready to eat beef Doner kebab during storage. Meat Sci. 2020, 169, 108217. [CrossRef]

21. Wang, J.; Jin, G.; Zhang, W.; Ahn, D.U.; Zhang, J. Effect of curing salt content on lipid oxidation and volatile flavour compounds of dry-cured turkey ham. Lwt-Food Sci. Technol. 2012, 48, 102-106. [CrossRef]

22. Jin, G.; He, L.; Zhang, J.; Xiang, Y.; Wang, J.; Feng, H. Effects of temperature and NaCl percentage on lipid oxidation in pork muscle and exploration of the controlling method using response surface methodology (RSM). Food Chem. 2012, 131, 817-825. [CrossRef]

23. Zhu, W.; Jiang, B.; Zhong, F.; Chen, J.; Zhang, T. Effect of Microbial Fermentation on the Fishy-Odor Compounds in Kelp (Laminaria japonica). Foods 2021, 10, 2532. [CrossRef] 
24. Jiang, X. Comparison of the Primary Odorants of Beijing Ducks with that of Cherry Valley Ducks. Food Ferment. Ind. 2004, 1, 21-24.

25. Toldrá, F.; Flores, M. The role of muscle proteases and lipases in flavor development during the processing of dry-cured ham. Crit. Rev. Food Sci. Nutr. 1998, 38, 331-352. [CrossRef]

26. Mathieu, S.; Cin, V.D.; Fei, Z.; Li, H.; Bliss, P.; Taylor, M.G.; Klee, H.J.; Tieman, D.M. Flavour compounds in tomato fruits: Identification of loci and potential pathways affecting volatile composition. J. Exp. Bot. 2009, 60, 325-337. [CrossRef]

27. Felipe, L.; Paulino, B.N.; Sales, A.; Molina, G.; Bicas, J.L. Chapter 15: Production of Food Aroma Compounds (Microbial and Enzymatic Methodologies). In Food Aroma Evolution; CRC Press: Boca Raton, FL, USA, 2019; pp. 293-306.

28. Allen, J.G.; Flanigan, S.S.; Mallory, L.B.; Jose, V.; Piers, M.N.; Stewart, J.H.; Christiani, D.C. Flavoring Chemicals in E-Cigarettes: Diacetyl, 2,3-Pentanedione, and Acetoin in a Sample of 51 Products, Including Fruit-, Candy-, and Cocktail-Flavored E-Cigarettes. Environ. Health Perspect. 2016, 124, 733-739. [CrossRef] [PubMed]

29. Figueira, J.A.; Porto-Figueira, P.; Pereira, J.; Cmara, J.S. Tangerines Cultivated on Madeira Island-A High Throughput Natural Source of Bioactive Compounds. Foods 2020, 9, 1470. [CrossRef] [PubMed]

30. Bittante, G.; Ni, Q.; Khomenko, I.; Gallo, L.; Biasioli, F. Rapid Profiling of the Volatilome of Cooked Meat by PTR-ToF-MS: Underlying Latent Explanatory Factors. Foods 2020, 9, 1738. [CrossRef]

31. Arce, L.; Gallegos, J.; Garrido-Delgado, R.; M Ed Ina, L.M.; Sielemann, S.; Wortelmann, T. Ion Mobility Spectrometry a Versatile Analytical Tool for Metabolomics Applications in Food Science. Curr. Metab. 2014, 2, 264-271. [CrossRef]

32. Li, F.; Feng, X.; Zhang, D.; Li, C.; Liu, Y. Physical properties, compositions and volatile profiles of Chinese dry-cured hams from different regions. J. Food Meas. Charact. 2020, 14, 492-504. [CrossRef]

33. Guo, Y.; Chen, D.; Ju, H.; Wu, C.; Lin, S. Characteristic volatiles fingerprints and changes of volatile compounds in fresh and dried Tricholoma matsutake Singer by HS-GC-IMS and HS-SPME-GC-MS. J. Chromatogr. B 2018, 1099, 46-55. [CrossRef]

34. Hy, A.; Wei, G.A.; Tong, X.A.; La, B.; Ht, A.; Chen, C.A. Aroma characteristics of traditional Huangjiu produced around Winter Solstice revealed by sensory evaluation, gas chromatography-mass spectrometry and gas chromatography-ion mobility spectrometry. Food Res. Int. 2021, 145, 110421. [CrossRef]

35. Fan, X.; Jiao, X.; Liu, J.; Jia, M.; Blanchard, C.; Zhou, Z. Characterizing the volatile compounds of different sorghum cultivars by both GC-MS and HS-GC-IMS. Food Res. Int. 2021, 140, 109975. [CrossRef] [PubMed]

36. Zeng, X.; Liu, J.; Dong, H.; Bai, W.; Yu, L.; Li, X. Variations of volatile flavour compounds in Cordyceps militaris chicken soup after enzymolysis pretreatment by SPME combined with GC-MS, GC x GC-TOF MS and GC-IMS. Int. J. Food Sci. Technol. 2020, 55, 509-516. [CrossRef]

37. Zhang, K.; Zhang, C.; Gao, L.; Zhuang, H.; Feng, T.; Xu, G. Analysis of volatile flavor compounds of green wheat under different treatments by GC-MS and GC-IMS. J. Food Biochem. 2021, e13875. [CrossRef]

38. Zhao, Y.; Zhan, P.; Tian, H.-L.; Wang, P.; Lu, C.; Tian, P.; Zhang, Y.-Y. Insights into the Aroma Profile in Three Kiwifruit Varieties by HS-SPME-GC-MS and GC-IMS Coupled with DSA. Food Anal. Methods 2021, 14, 1033-1042. [CrossRef]

39. Elmore, J.S.; Mottram, D.S.; Enser, M.; Wood, J.D. Effect of the polyunsaturated fatty acid composition of beef muscle on the profile of aroma volatiles. J. Agric. Food Chem. 1999, 47, 1619. [CrossRef] [PubMed]

40. Marui, N.; Petrovi, M.; Vi Da Ek, S.; Petrak, T.; Medi, H. Characterization of traditional Istrian dry-cured ham by means of physical and chemical analyses and volatile compounds. Meat Sci. 2011, 88, 786-790.

41. Lorenzo, J.M.; Carballo, J.; Franco, D. Effect of the Inclusion of Chestnut in the Finishing Diet on Volatile Compounds of Dry-Cured Ham from Celta Pig Breed. J. Integr. Agric. 2013, 12, 2002-2012. [CrossRef]

42. Giri, A.; Osako, K.; Ohshima, T. Identification and characterisation of headspace volatiles of fish miso, a Japanese fish meat based fermented paste, with special emphasis on effect of fish species and meat washing. Food Chem. 2010, 120, 621-631. [CrossRef]

43. Martinez-Onandi, N.; Rivas-Canedo, A.; Avila, M.; Garde, S.; Nunez, M.; Picon, A. Influence of physicochemical characteristics and high pressure processing on the volatile fraction of Iberian dry-cured ham. Meat Sci. 2017, 131, 40-47. [CrossRef] [PubMed]

44. Edwards, R.A.; Ordonez, J.A.; Dainty, R.H. Characterization of the headspace volatile compounds of selected Spanish dry fermented sausages. Food Chem. 1999, 64, 461-465. [CrossRef]

45. Wang, L.; Gao, M.; Liu, Z.; Chen, S.; Xu, Y. Three Extraction Methods in Combination with GC $\times$ GC-TOFMS for the Detailed Investigation of Volatiles in Chinese Herbaceous Aroma-Type Baijiu. Molecules 2020, 25, 4429. [CrossRef] [PubMed] 